\title{
Miniaturized On Board Data Acquisition Unit
}

\author{
Güven Solak', Tuğba Çelebí, Onur Yașar ${ }^{3}$ \\ ${ }^{1}$ Aselsan Inc., Turkey, gsolak@aselsan.com.tr, \\ ${ }^{2}$ Aselsan Inc., Turkey, tsiltu@aselsan.com.tr. \\ ${ }^{3}$ Aselsan Inc., Turkey, onuryasar@aselsan.com.tr
}

\begin{abstract}
:
In today's world as smart munitions become increasingly complex, it became mandatory to monitor the activity of munitions during launch and flight by means of gathering critical operational data. This critical data is also very important to reduce the development cost, development time and failure investigation effort. The most challenging part of this study is that the instrument developed for monitoring munitions' activity must withstand very harsh environments that munitions experience.

Since existing data acquisition systems are too big to fit inside newly developed munitions, a miniature and shock hardened on board data acquisition unit is developed. The unit acquires several analog and digital channels and has an SPI interface for on board recording or real time transmitting. This unit is successfully operated and flight data is collected.

In this paper, the properties of the data acquisition unit such as size, analog and digital channels, data storage capacity and data recording and transmitting algorithm are explained and test results are presented.
\end{abstract}

Key words: On Board Data Acquisition, Smart Munitions, On Board Recording

\section{Introduction}

During development and design verification phases of smart munitions, it is of upmost importance to gather realistic data related to the components that constitute the fuze. For this purpose, a g-hardened and miniature on board data acquisition unit has been developed. The data gathered by data acquisition unit can be recorded to the memory on board or can be transmitted by means of a telemetry link. In this paper the data acquisition hardware, data recording and transmitting algorithms are described and experimental data obtained from projectile flight tests that made use of this hardware are presented.

\section{Miniaturized On Board Data Acquisition Hardware}

The main function of the on board data acquisition unit is to collect data during flight and launch of a projectile and than record that data for recovery after flight or transmit through a telemetry link. The hardware architecture is mainly based on an ultra low power microcontroller. There are six analog and five digital channels on the unit. As an addition there is an SPI interface and serial interface. The SPI interface is used to record the collected data on the flash memory or to interface to the transceiver IC for online transmitting. The serial interface is for easy play backing of the recorded data. There is and $A / D$ converter that have a conversion rate of $1 \mathrm{Msps}$ for fast changing analog channels. The unit can sample and record data at a rate of 24000 samples per second with 8 bit resolution. The memory is 32 Mbits size. The unit is triggered by an external trigger. The diameter of the unit is $22 \mathrm{~mm}$. The unit is implemented in rigid flex form as illustrated in Fig. 1. 


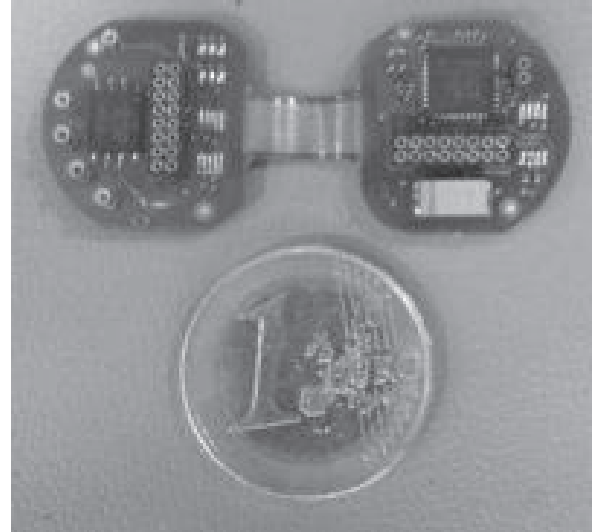

Fig.1. The data acquisition unit

Using rigid flex technology made possible to minimize the size of the unit and to use more circuit elements in a condensed volume. The unit had experienced acceleration loads at least $60000 \mathrm{~g}$ and still survived after such high acceleration loads.

\section{Data Recording and Transmitting Algorithm}

The software running on the data acquisition unit varies according to the test that will be implemented. Mainly, the core scenario of the software remains the same but the analog channels that will be sampled, the sample rate for the analog channels may vary. The start scenario of the data acquisition unit is determined according to the feedback taken from the activities carried out before firing. The unit waits for an internal trigger in low power mode. After the trigger, the data acquisition activities begin. The data collected are buffered for storing on the flash memory or for real time transmitting.
Since the memory that the data are stored and the power source used is limited, there are precautions for not filling the memory before storing the most important data under interest. In order to suffer from this, for some signals, the unit only detects a significant change in state and store that valuable data with an appropriate time stamp.

For transmitting through a telemetry link, the unit buffers the acquired data and then transmits as soon as possible the projectile lefts the cannon. In order not to suffer from power disconnections during firing, the unit holds a key variable and decides if it began functioning before or not. By this way in case of any power discontinuity, it resumes from where it left.

\section{Test Results}

Several tests are carried out in order to see if the data acquisition unit can withstand harsh firing environments. For this purpose, shock hardened units are assembled to the projectiles that simulate the interior ballistics of the projectiles [1] that are being developed. In these tests several signals such as the power supply voltage of the unit, three axis accelerometer data and a firing indicator are sampled. For the tests that the data are stored on board memory, the projectile is softly recovered and flight data are successfully downloaded to the PC. Fig.2. shows the acceleration data collected during launch of a projectile. This data is play backed after the projectile recovery. Fig.3. shows the acceleration data downloaded with a telemetry link. In both of these tests the data acquisition unit and the accelerometer successfully gun launch and projectile flight.

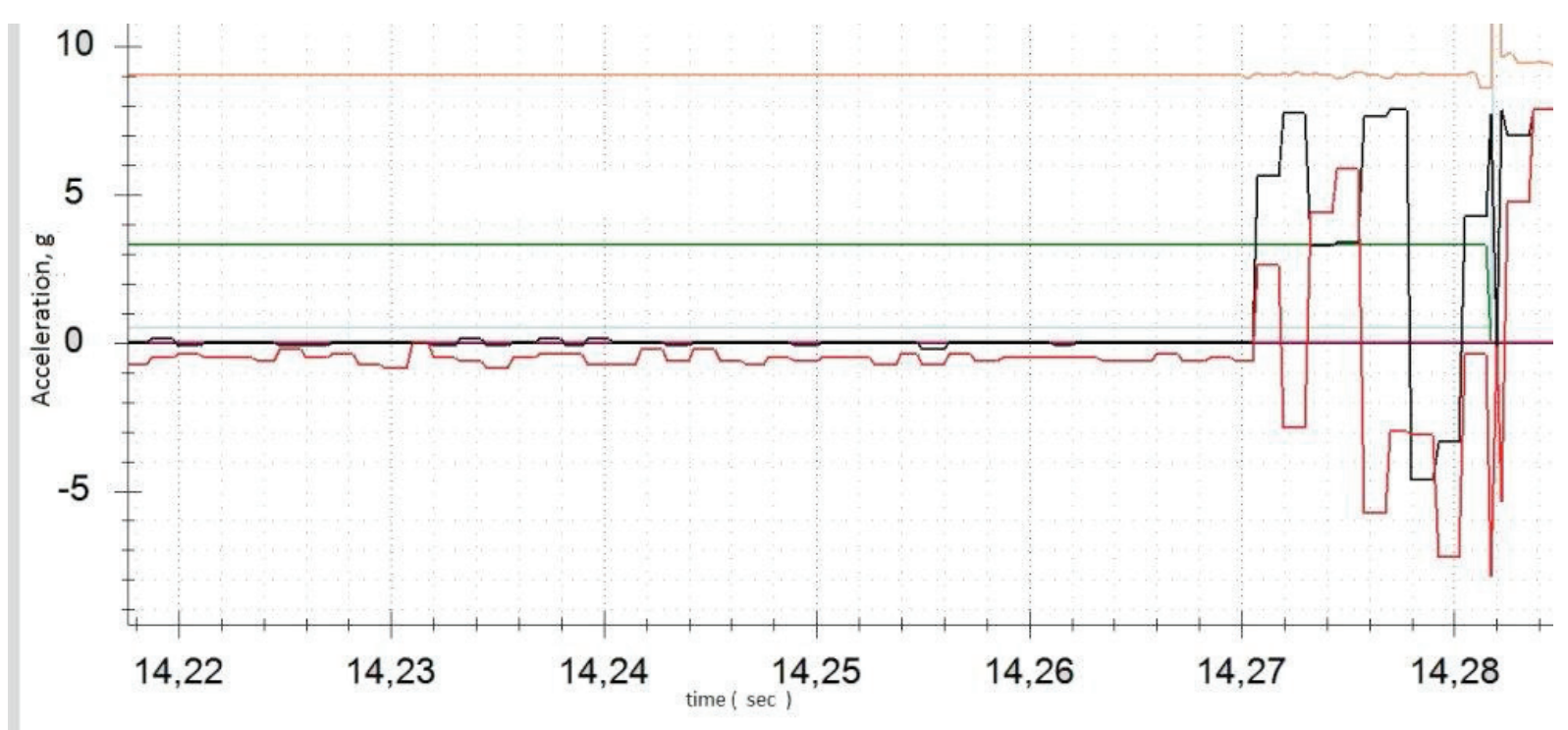


Fig.2. Acceleration data acquired with data acquisition unit. This data is played back from the unit after soft recovery.

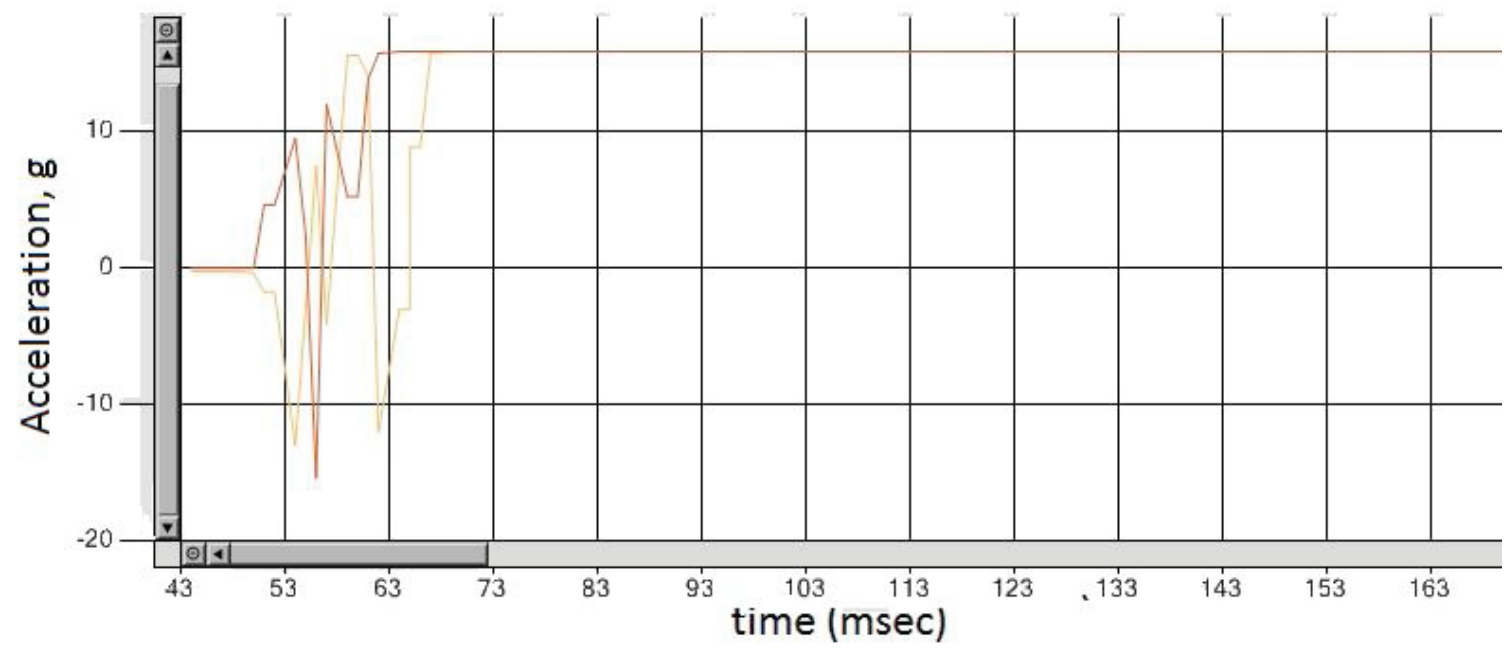

Fig.3.Acceleration data acquired with data acquisition unit. This data is downloaded by means of a telemetry link

\section{Conclusions}

In this study, an on board data acquisition unit is developed and implemented. Several flight tests proved that the data acquisition unit survives after projectile launch and impact. The flight data is successfully gathered by means of play backing the data stored on an onboard memory or downloading through a telemetry link. This provided an effective and low cost method for testing activities that will be of at upmost importance in emerging munitions development concepts.

\section{References}

[1] M. Akcay, Balistik 1993 